\title{
The need for evidence-based intake, progress, and outcomes assessment
}

\author{
Thomas M. Achenbach ${ }^{1}$
}

Published online: 9 December 2016

(C) Springer-Verlag Berlin Heidelberg 2016

Mental health clinicians are under increasing pressure to provide evidence for the effectiveness of their services. Many kinds of treatment are being promoted as "evidence based," with randomized controlled trials (RCTs) serving as gold standards for evidence-based treatments. Despite the importance of RCTs for evaluating treatments, it has been found that therapeutic benefits achieved in RCTs often shrink markedly when the treatments are applied to children in community mental health services $[1,2]$.

One possible reason for the shrinkage of benefits is that children seen in community services are not recruited for having the specific problems targeted by particular evidence-based treatments. A second possible reason for shrinkage is that community clinicians are not trained to provide all the relevant evidence-based treatments. And a third possible reason is that parents in community services may not collaborate as effectively as parents participating in RCTs.

\section{Whether to treat, what to treat, whom to treat?}

To benefit from RCT findings, clinicians need to have a clear basis for determining whether particular children need treatment. If treatment is indicated, clinicians also need a clear basis for determining what should be treated and who should be treated, whether it should be the child, one parent, both parents, or all of them. To aid clinicians in deciding whether to treat, what to treat, and whom to treat, evidence-based assessment instruments are needed to obtain

Thomas M. Achenbach

thomas.achenbach@med.uvm.edu

1 Burlington, USA standardized data on problems and strengths manifested by the child and parents. Parallel self-report and collateralreport instruments for developmentally appropriate assessment of child and parent functioning can give clinicians a comprehensive picture of areas in which children and parents may need help [3]. To qualify as evidence-based, assessment instruments should be supported by published standardization, reliability, validity, and normative data [4].

\section{Multi-informant dimensional assessment of children and their parents}

Assessment instruments that are scored on developmentally normed dimensional scales document the problems and strengths that can then be compared with subsequent reassessments to measure the progress and outcomes of treatment. If clinicians deem it appropriate, they can show parents bar graphs of dimensional scales that are scored from ratings of their child's functioning, their partner's functioning, and their own functioning. Bar graphs of data from multiple informants can help parents understand how views of their child's functioning and of their own functioning may differ. For example, a child's teachers may report more attention problems than reported by the child or parents. On the other hand, a child may report more depression than reported by the child's parents or teachers. One parent may report that the other parent displays much more aggressive behavior than that parent reports. Graphs of normed dimensional scores derived from each informant's reports provide clinicians and parents with concrete pictures of problems for which help may be needed. Graphs also reveal differences between informants' reports that can help parents understand variations in how their child functions in different contexts and in how their child is seen by different informants. 


\section{How and how much to treat?}

In addition to helping clinicians decide whether to treat, what to treat, and whom to treat, dimensional scales can help clinicians decide how and how much to treat. Very high problem scores would argue for high-intensity treatment, such as high doses of medication, frequent therapy sessions, or inpatient or residential placements. Lower problem scores would argue for lower intensity treatment or other kinds of treatment. Depending on scores obtained by the child and parents, the results might argue for lowintensity treatment for the child but higher intensity treatment for one or both parents.

\section{How long to treat?}

The benefits of evidence-based assessment are not limited to the initial phases of services. Because evidence-based assessment quantitatively documents problems and strengths, re-administration of the same assessment instruments during the course of treatment provides updated scores for the child and parents that can be compared with previous scores. Software for comparing earlier and later scores can produce text stating whether changes exceed chance expectations [5]. The software uses statistical criteria to determine whether changes exceed chance expectations, but clinicians and parents do not need statistical knowledge to understand the text statements about whether changes exceed chance. The information about changes can help clinicians and parents decide whether to continue, modify, or end the treatment. Multiple studies have found that progress assessments improve the outcomes of mental health services [6-8].

\section{How good are treatment outcomes?}

Much of the pressure on clinicians to provide evidence for effectiveness focuses on outcomes. To determine whether outcomes are favorable, it is essential to compare children's outcome functioning with their initial functioning. Evidencebased dimensional assessment enables clinicians to determine whether improvements exceed chance expectations by using software that measures changes from initial scale scores to outcome scores. As done for comparisons between initial and progress assessments, computer-generated text states whether changes from initial to outcome assessments exceed chance expectations. Equally important, norms for dimensional scales tell clinicians whether a child's scores have reached the normal range. If scores are not in or close to the normal range, clinicians may elect to continue or augment treatment until the scores approximate the normal range.
It is important to ascertain whether improvements are maintained. This can be done by performing follow-up assessments at periods such as 6 and 12 months after termination. By having the same assessment instruments completed at follow-ups, clinicians can determine whether changes since intake exceed chance and whether follow-up scores are in the normal range.

\section{Research applications}

Thus far, I have focused on the value of dimensional, evidence-based assessment for individual children and their parents. However, such assessment is equally valuable in clinical research. To measure the effects of treatments, it is essential for participants to be assessed with the same dimensional methods before and after treatment. To track the course of change, it is desirable to assess participants at one or more points during the course of treatment. In the current ECAP, this point is highlighted by Skarphedinsson et al. [9], who used the Children's Yale-Brown Obsessive-Compulsive Scale (CY-BOCS) to assess Nordic children before, during, and after cognitive-behavioral therapy for obsessive-compulsive disorders. CY-BOCS scores obtained from independent evaluators were plotted against Clinical Global Impression ratings by clinicians to identify optimal cutoff scores for defining favorable treatment responses and remission of OCD disorders.

As the authors state, "It is important to standardize treatment outcome criteria so as to facilitate comparisons across trials and establishment of treatment guidelines... cutoffs can assist in decision-making... by informing whether to switch or augment treatment in the absence of response, continue treatment... or provide booster sessions."

In another article in the current ECAP, Benarous et al. [10] reviewed treatments for youths with severely dysregulated mood. It was found that nearly all the studies were limited by a lack of standardized evidence-based assessment of treatment effects. Considering the formidable treatment challenges posed by severely dysregulated mood, it is essential that evidence-based assessment instruments be used to measure outcomes and to provide generalizable data.

\section{References}

1. Weisz JR, Krumholz LS, Santucci L, Thomassin K, Ng MY (2015) Shrinking the gap between research and practice: tailoring and testing youth psychotherapies in clinical care contexts. Annu Rev Clin Psychol 11:139-163. doi:10.1146/ annurev-clinpsy-032814-112820

2. Weisz JR, Ugueto AM, Cheron DM, Herren J (2013) Evidencebased youth psychotherapy in the mental health ecosystem. J 
Clin Child Adolesc Psychol 42:274-286. doi:10.1080/15374416. 2013.764824

3. Achenbach TM, Rescorla LA, Ivanova MY (2015) Guide to family assessment using the ASEBA. University of Vermont Research Center for Children, Youth, and Families, Burlington

4. Hunsley J, Mash EJ (eds) (2017) A guide to assessments that work, 2nd edn. Oxford University Press, New York

5. Achenbach TM (2017) Manual for assessing progress and outcomes in problems and strengths. University of Vermont Research Center for Children, Youth, and Families, Burlington

6. Bickman L, Kelley SD, Breda C, de Andrade AR, Riemer M (2011) Effects of routine feedback to clinicians on mental health outcomes of youths: results of a randomized trial. Psychiatr Serv 62:1423-1429. doi:10.1176/appi.ps.002052011

7. Simon W, Lambert MJ, Harris MW, Busath G, Vazquez A (2012) Providing patient progress information and clinical support tools to therapists: effects on patients at risk of treatment failure. Psychother Res 22:638-647. doi:10.1080/10503307.201 2.698918

8. Shimokawa K, Lambert MJ, Smart DW (2010) Enhancing treatment outcome of patients at risk of treatment failure: meta-analytic and mega-analytic review of a psychotherapy quality assurance system. J Consult Clin Psychol 78:298-311. doi:10.1037/ a0019247

9. Skarphedinsson G, De Nadai AS, Storch EA, Lewin AB, Ivarsson T (2017) Defining cognitive-behavior therapy response and remission in pediatric OCD: a signal detection analysis of the Children's Yale-Brown Obsessive-Compulsive Scale. Eur Child Adolesc Psychiatry. doi:10.1007/s00787-016-0863-0

10. Benarous X, Consoli A, Guilé JM, Garny de La Rivière S, Cohen D, Olliac B (2017) Evidence-based treatments for youths with severely dysregulated mood: a qualitative systematic review of trials for SMD and DMDD. Eur Child Adolesc Psychiatry. doi:10.1007/s00787-016-0907-5 